
\title{
3 Research Square \\ Independent control of amplitude and period in the synthetic repressilator
}

Fengyu Zhang

Peking University https://orcid.org/0000-0002-9196-3042

\section{Yanhong Sun}

Peking University

\section{Yihao Zhang}

Peking University https://orcid.org/0000-0002-1581-5937

\section{Wenting Shen}

Peking University

\section{Shujing Wang}

Peking University

\section{Qi Ouyang}

Peking University

Chunxiong Luo ( $\sim$ pkuluocx@pku.edu.cn )

Peking University https://orcid.org/0000-0001-8974-7693

\section{Article}

Keywords: synthetic oscillator circuit, repressilator, orthogonal

Posted Date: June 11th, 2021

DOI: https://doi.org/10.21203/rs.3.rs-584751/v1

License: (c) (i) This work is licensed under a Creative Commons Attribution 4.0 International License.

Read Full License

Version of Record: A version of this preprint was published at Communications Biology on January 11th, 2022. See the published version at https://doi.org/10.1038/s42003-021-02987-1. 


\title{
Independent control of amplitude and period in the synthetic repressilator
}

\author{
Fengyu Zhang ${ }^{1,2}$, Yanhong Sun ${ }^{2}$, Yihao Zhang ${ }^{1,3}$, Wenting Shen ${ }^{2,3}$, Shujing Wang ${ }^{2,3}$, \\ Qi Ouyang ${ }^{3}$, Chunxiong Luo Lu, $^{2,4,5 *}$
}

\author{
${ }^{1}$ School of Life Sciences and Peking-Tsinghua Center for Life Sciences, Peking University, Beijing \\ 100871, China. \\ ${ }^{2}$ The State Key Laboratory for Artificial Microstructures and Mesoscopic Physics, School of \\ Physics, Peking University, China; \\ ${ }^{3}$ Center for Quantitative Biology, Academy for Advanced Interdisciplinary Studies, Peking \\ University, China. \\ ${ }^{4}$ Wenzhou Institute University of Chinese Academy of Sciences, Wenzhou, Zhejiang, China; \\ ${ }^{5}$ Oujiang Laboratory, Wenzhou, Zhejiang, China \\ *To whom correspondence should be addressed: Chunxiong Luo, email: pkuluocx@pku.edu.cn.
}

\begin{abstract}
Synthetic Biology aims to create predictable biological circuits and fully operational biological systems. Although there are methods to create more stable oscillators, such as repressilators, orthogonally controlling the oscillation of reporter genes in terms of their amplitude and period is only on theoretical level. Here, we introduce a new oscillator circuit that can be orthogonally controlled by two inducers in Escherichia coli. Some control components, including $\sigma \mathrm{ECF} 11$ and NahR, were added to the circuit. By systematically tuning the concentration of the inducers, salicylate and IPTG, the amplitude and period can be modulated independently. Furthermore, we constructed a quantitative model to forecast the regulation results. Under the guidance of the model, the expected oscillation can be regulated by choosing the proper concentration combinations of inducers. In summary, our work achieved orthogonal control of the oscillator circuit, which allows the oscillator to be modularized and used in more complex circuit designs.
\end{abstract}

Key words : synthetic oscillator circuit, repressilator, orthogonal 


\section{Introduction}

Many important physiological processes are controlled by oscillatory signals, such as the P53 protein, cell cycles [1][2] and biological circadian rhythms [3][4]. Via complex control circuits [5], organisms can achieve stable oscillations and adjust the correlation parameters - period, amplitude and phase-accurately. Compared to these natural cyclic phenomena, synthetic oscillations are difficult to control in such complex networks; thus, stable and accurate control of oscillation is a problem. Recently, synthetic biology has sought to make oscillations robust and tunable in two kinds of synthetic oscillators: the dual-feedback oscillator (DFO) [6] and repressilator (RLT) [7]. For the DFO, some amount of control has been achieved using two changed inducer concentrations [8] or extra control components [9]. However, the orthogonal control of the RLT, which was developed earlier and is more widely researched than the DFO, has still not been realized. Two recent studies have reported significant progress: improvement of stability [10] and the theoretical direction of orthogonal control [11]. These works set the stage for orthogonally controlling repressilators experimentally.

Based on its general principle, that is, a negative feedback loop with a time-delay [12], the repressilator is constructed of three transcriptional repressors acting in sequence [7]. The three transcriptional repressors contain TetR from the Tn10 transposon, cI from bacteriophage $\lambda$ and LacI from the lactose operon. In a period of oscillation, each repressor inhibits the transcription of the next one (a-b-c-a), which gives a time delay and leads to the oscillation. Because this is a complete synthetic circuit unlike those in the natural world, it is difficult to control the oscillation in the absence of a reference.

To solve this problem, we used the strategy of a dual-input promoter [13] to accomplish orthogonal control of amplitude and period experimentally referring to Tomazou's theoretical work [11]. Two kinds of inducers were used to control the reporting protein node and one oscillation node independently. The accuracy of the control was demonstrated using a microfluidic system [14], and quantitative analysis models were established. Through our proposed quantitative model, the oscillations under a particular combination of inducer concentrations can be predicted; thus, we can 
choose appropriate inducer combinations to achieve the expected effect. Our work developed the theory of the orthogonal control of the repressilator in practice, which may give some guidance to realize an adjustable repressilator experimentally and make some progress in the quantification and modularization of repressilators.

\section{Results}

\section{Constructing the gene circuits}

On the basis of our design strategy to orthogonal control of amplitude and period of repressilator, we developed a gene circuit construction from the pLPT107 and pLPT41 plasmids used in Potvin`s work[10] (Fig. S1). The original pLPT107 plasmid had the original repressilator and an mVenus fluorescent protein gene which act as a reporter. It also had a constant-expressed CFP gene as a marker, which was deleted in our subsequent design. The original pLPT41 plasmid only had no functional genes except two binding sites of TetR.

To accomplish the orthogonal regulation of amplitude and period, the most important step is the introduction of the dual-input promoter. To change the promoter of the reporter gene, mVenus, we constructed a subcloning containing the upstream and downstream gene sequence of the original promoter, which was a pLTetO-1 promoter. Then the dual promoter, which was developed from the promoter of $\sigma E C F 11[15]$, was added into the subcloning by repeatedly PCR steps. The dual promoter had the binding site of both $\sigma \mathrm{ECF} 11$ and TetR, so it can be regulated by these two regulators and the regulation was orthogonal[13]. Then the subcloning was integrated into the original pLPT107 plasmid by gibson assembly.

On the other hand, the control system of $\sigma E C F 11$ was introduced into the pLPT41 plasmid. The $\sigma E C F 11$ gene was designed to be regulated by an NahR-pSal system[16][17]. The $\sigma E C F 11$ gene, the $N a h R$ gene and relevant promoters were all constructed into the pLPT41 plasmid by gibson assembly(Fig.1a).

Through the reconstructions mentioned above, we can realize the independent regulation of the repressilation. The expression of $\sigma \mathrm{ECF} 11$, which controlled by NahR$p S a l$ system, can lead to the regulation of amplitude. The inducer salicylate $\left(\mathrm{I}_{1}\right)$ can 
relieve the repress of NahR so that the quantity of $\sigma \mathrm{ECF} 11$, which decided the amplitude, was related to the salicylate concentration. As for the period, we used IPTG(I2) as the input, which can weaken the repression of LacI. Thus the status switching will be slower but the oscillation can still keep within limits, which lead to a regulation of period (Fig.1b).

\section{Constructing the observation system}

To observe an oscillation at the single cell level, we used the "mother machine" microfluidic system. We designed 8 main channels on a chip, and each channel had 120 growth channels (Fig. 1c). The main channels were designed to be $100 \mu \mathrm{m}$ width and $20 \mu \mathrm{m}$ height. The growth channels were designed to be about $1.5 \mu \mathrm{m}$ width, $1.5 \mu \mathrm{m}$ height and $30 \mu \mathrm{m}$ in length. The growth channels were on the same side of the main channels which could easily load the bacteria through centrifugal force. Using this device, 8 different combinations of inducer concentrations could be preformed in one experiment. In the mother machine, cells were trapped and grew in the growth channel with the continuously replacement of medium in the main channel (Fig. 1d). The medium was pumped at a speed of $40 \mu \mathrm{L} / \mathrm{h}$ by a syringe pump, which is an appropriate speed to guarantee the nutritional supply for the growth of bacteria and stability of the chip. 
a

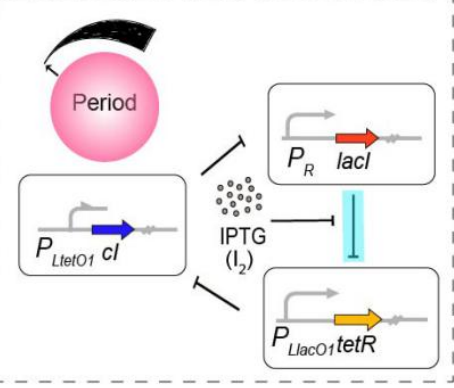

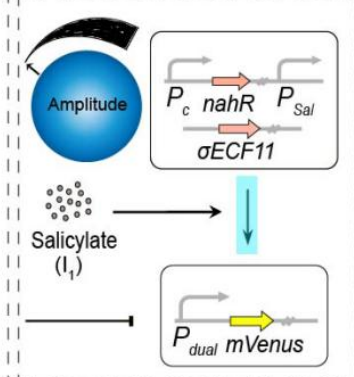

b

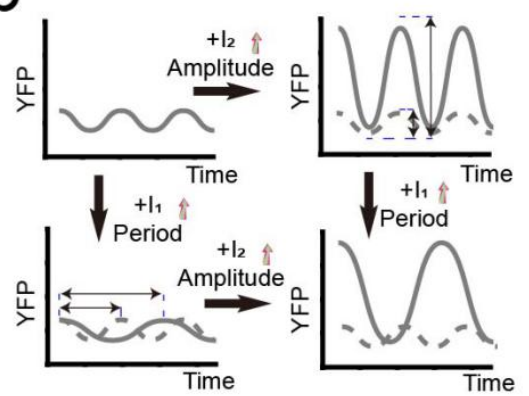

C

Media inlets Cell Loading and Waste

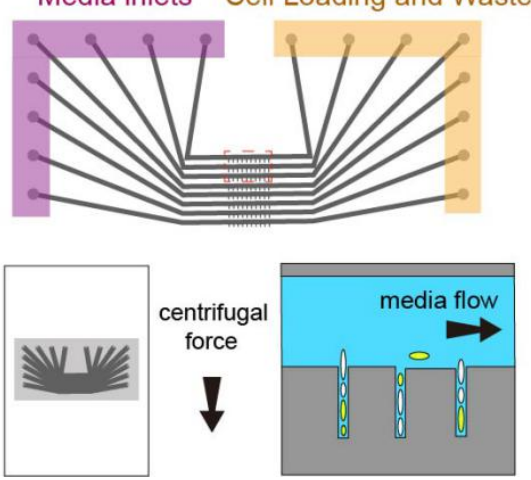

d

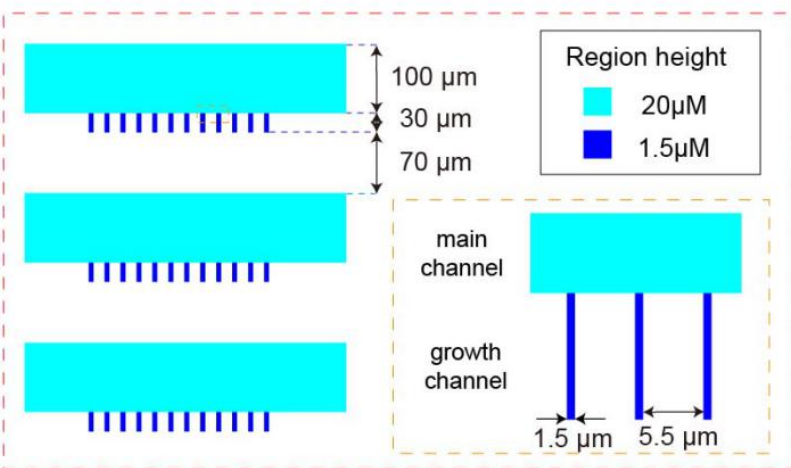

Figure 1. Construction and observation of the independent regulation of the repressilator. a.

Construction of the gene circuits. The mVenus gene, which act as a reporter, can be regulated by a dual-input promoter. Salicylate can regulate the expression level of the mVenus gene, which leads to a change in amplitude. IPTG can regulate the time of the state switch, which leads to a change in period. b. Schematic diagram of the orthogonal regulation. Input $\mathrm{I}_{1}$ regulates the period and Input $I_{2}$ regulates the amplitude independently. The combination of $I_{1}$ and $I_{2}$ can lead to an oscillation with an expected amplitude and period. c. Design of the mother machine microfluidic chips to observe the repressilator under different conditions. Eight combinations of different conditions can be observed in an experiment. d. Observation of the bacteria growing in the chip. Daughter cells are washed away when they grow over the border.

\section{Observing and tuning the oscillation}

To check the results of our design, the E.coli cells contained in the synthetic gene circuit were analyzed in the mother machine microfluidic system. An on/off test of the regulation of both amplitude and period was performed to check the feasibility of the inducer regulation and stability of the microfluidic system. For the regulation of amplitude, we set the concentration of IPTG to $0 \mu \mathrm{M}$ and chose two salicylate 
concentrations, 0 and $200 \mu \mathrm{M}$. The results showed that both the low and high concentration groups grew stably and underwent a complete period of oscillation. The regulation also worked. The fluorescence intensity amplitude, defined as the difference between a peak and the nearest previous trough, increased by approximately 100 times, generally after the addition of salicylate (from approximately 20 a.u. to approximately 2000 a.u.). Two typical channels were shown in Fig. 2a, which showed microscopic images of the whole channel over 15 hours.

Similarly, for the regulation of the period, we chose two IPTG concentrations, 0 and $10 \mu \mathrm{M}$ (the concentration of salicylate was set to $100 \mu \mathrm{M}$ ). It could be seen in the two typical channels (Fig. 2a) that the 0 IPTG concentration group had three peaks and the $10 \mu \mathrm{M}$ IPTG concentration group had only two peaks, which meant that the period of oscillation increased with the IPTG concentration.

a
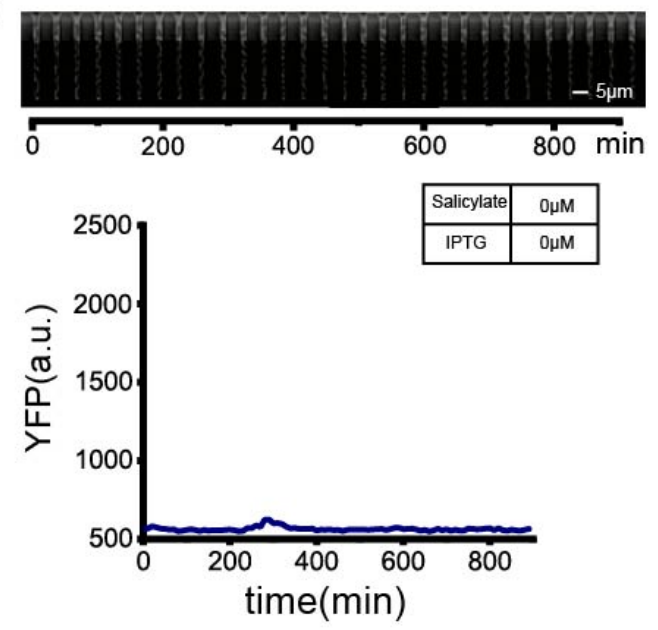

C
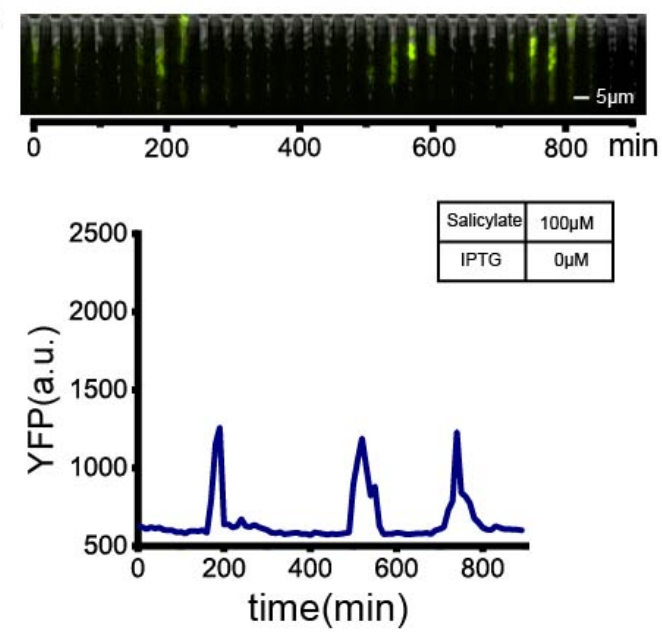

b
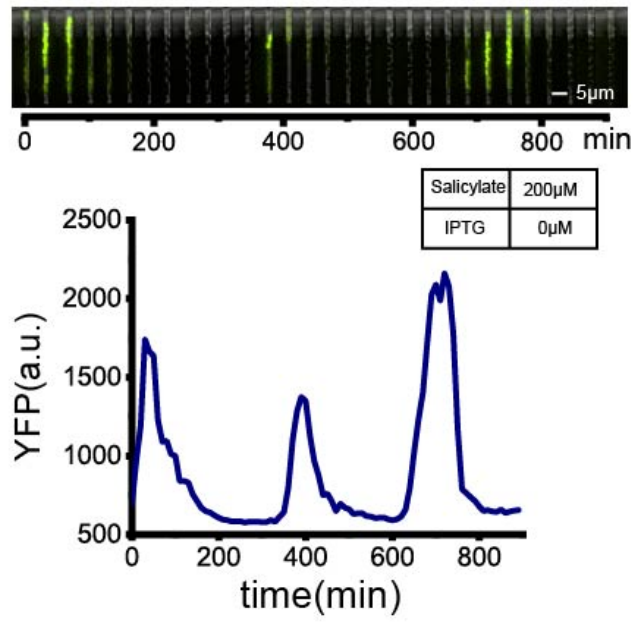

d
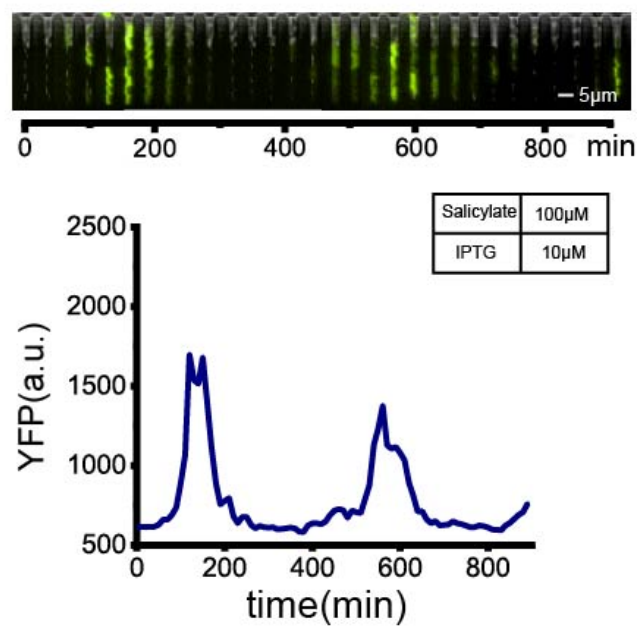

Figure 2. Microscope images and the single cell fluorescence intensity curve of the oscillation in different inducer concentration combinations (a. IPTG, $0 \mu \mathrm{M}$; salicylate, $0 \mu \mathrm{M}$, b. IPTG, $0 \mu \mathrm{M}$; 
salicylate, $200 \mu \mathrm{M}$, c. IPTG, $0 \mu \mathrm{M}$; salicylate, $100 \mu \mathrm{M}$, d. IPTG, $10 \mu \mathrm{M}$; salicylate, $100 \mu \mathrm{M}$ ). Microscope images were merged by the phase contrast and YFP channels. The single cell curves were drawn from the fluorescence intensity of the mother cell in these channels. Each curve has at least one complete period of oscillation.

To further confirm the independent regulation ability of the repressilator circuit, we performed a series of dose-response experiments. Totally $4 \times 4$ combinations of inducer concentrations were used in the amplitude and period regulation. The concentration of IPTG had a gradient of $0,2.5,5,10 \mu \mathrm{M}$ and the salicylate had a gradient of $0,50,100,200 \mu \mathrm{M}$. The experiments showed the similar results as the on/off test. 5 typical fluorescence intensity curves of mother cells for each group were shown in Fig. S2.

The statistical results of the dose-response experiments were shown in Fig. 3. The results were shown in two forms, which represented the independent regulation of amplitude and the independent regulation respectively. Fig. 3a represented the independent regulation of amplitude. It can be seen that in all of the four IPTG concentration groups, which were shown as four curves with different colors, the cells that received different concentrations of salicylate had diverse amplitudes, but the periods were similar. Thus, we realized independent regulation of amplitude via $I_{1}$, salicylate. Similarly, Fig. 3 b represented the independent regulation of period. From the results shown in Fig. 3b, we can see that the amplitudes were similar but the periods were diverse among cells that received different concentrations of IPTG, which satisfied our design. From the results of these experiments, we can state that orthogonal regulation of repressilator was realized at the experimental level. 
a

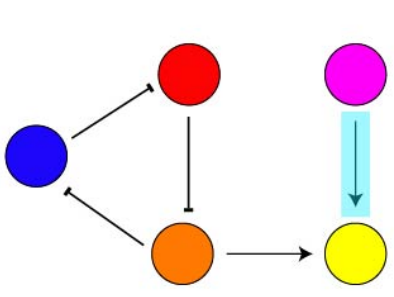

b

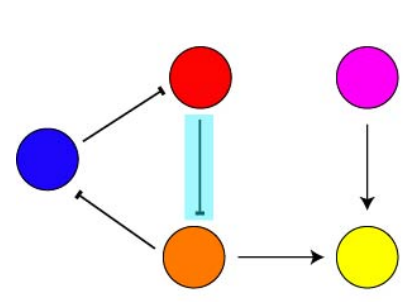

Independent Regulation of Amplitude
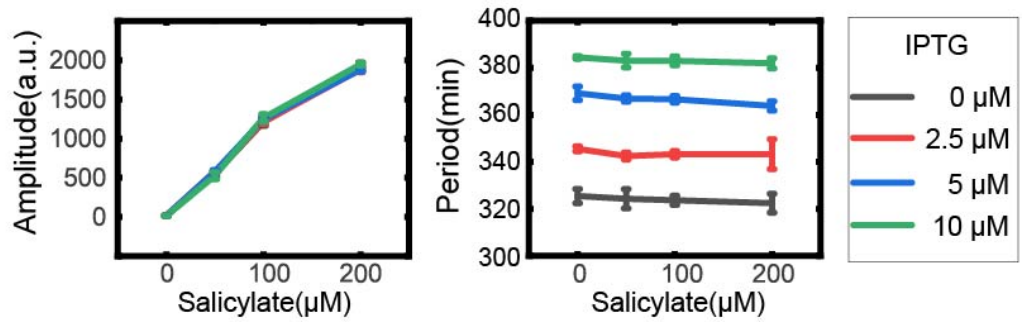

Independent Regulation of Period
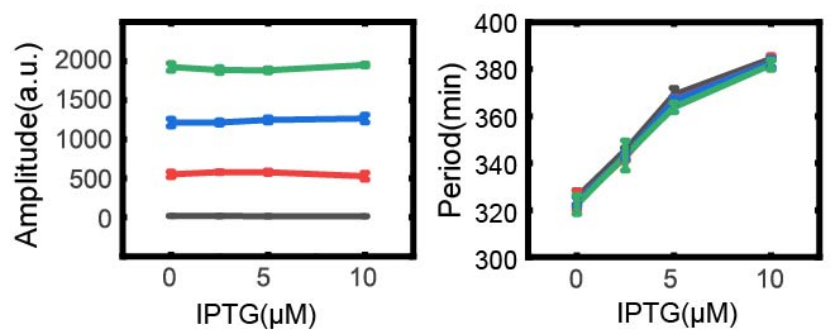

Salicylate

$-0 \mu \mathrm{M}$

$-50 \mu \mathrm{M}$

$-100 \mu \mathrm{M}$

$200 \mu \mathrm{M}$

Figure 3. Independent regulation of amplitude and period. a. Independent regulation of amplitude. 16 groups of single cells were shown as four curves vary with different salicylate concentrations. The four curves respectively represent the IPTG concentration of $0 \mu \mathrm{M}, 2.5 \mu \mathrm{M}, 5 \mu \mathrm{M}$ and $10 \mu \mathrm{M}$. b. Independent regulation of amplitude. The same 16 groups of single cells were shown as four curves vary with different IPTG concentrations. The four curves respectively represent the IPTG concentration of $0 \mu \mathrm{M}, 50 \mu \mathrm{M}, 100 \mu \mathrm{M}$ and $200 \mu \mathrm{M}$. Each group had the results of three independent experiments and each experiment had over 100 single cells. The data point were calculated from the average of the three experiments and the error bars were calculated from the SD of the average of the three experiments.

It is also worth mentioning that the orthogonal regulation was not without limits. The results of some additional experiments showed that the amplitude no longer raised with the increase of salicylate when the concentration was greater than $200 \mu \mathrm{M}$. And the period no longer raised with the increase of IPTG when the concentration was greater than $10 \mu \mathrm{M}$, either. This could be due to the saturation of the inducers. More detailed data were shown in Fig. S3.

\section{Constructing the quantitative model}


To systematically describe orthogonal regulation in development, we constructed a quantitative model. The model was mainly based on Tomazou's work [11] with some modifications. The model had three parts, as shown in Fig. 4a, which contained the regulation of period, amplitude and output.

First, gene expression follows a transitional transcription-translation model [11]. The relevant variables are as follows: the molecular number of functional proteins, which means they are already folded ( $R_{1}$ for $\lambda \mathrm{cI}, R_{2}$ for LacI, $R_{3}$ for TetR, $R_{4}$ for NahR, $E$ for $\sigma E C F 11, Y$ for mVenus); molecular number of unfolded proteins $\left(X_{u}, X=R_{1}, R_{2}\right.$, $\left.R_{3}, R_{4}, E, Y\right)$; molecular number of corresponding mRNAs $\left(m_{x}, x_{=} R_{1}, R_{2}, R_{3}, R_{4}, E, Y\right)$; translation rate constant $\left(r_{x}, x=R_{1}, R_{2}, R_{3}, R_{4}, E, Y\right)$; folding rate constant $\left(k_{f x}, x=R_{1}, R_{2}\right.$, $R 3, R 4, E, Y)$; and the protein dilution rate due to cell division $(\delta)$. Therefore, the equations for proteins are as follows:

$$
\begin{aligned}
& \frac{d X_{u}}{d t}=r_{x} m_{x}-k_{f x} X_{u}-\delta X_{u} \\
& \frac{d X}{d t}=k_{f x} X_{u}-\delta X \\
& \left(x=R_{i}(i=1,2,3,4), E, Y\right)
\end{aligned}
$$




\section{a}
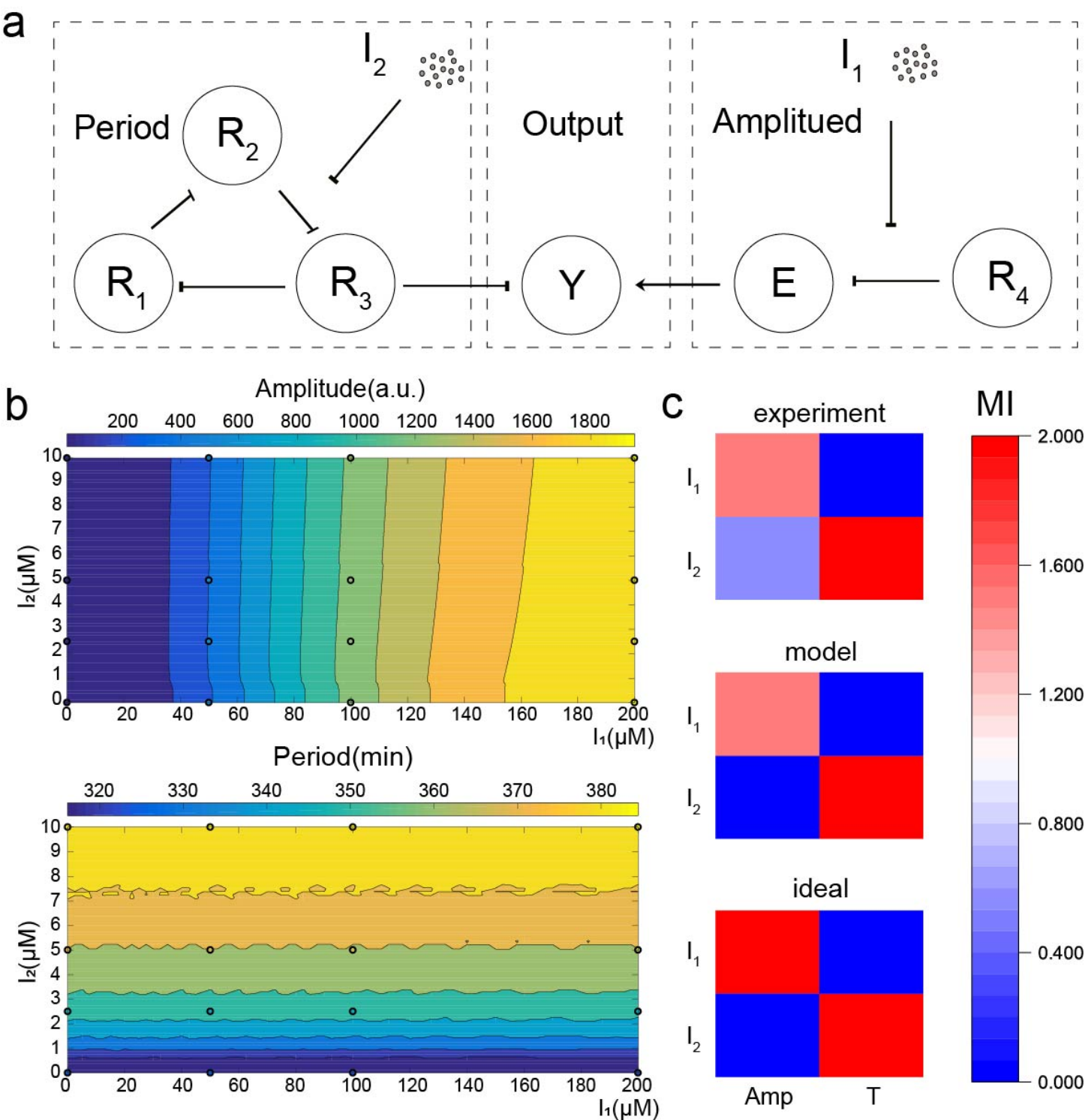

Figure 4. Quantitative mathematical modeling of the repressilator. a. Relation diagram of the quantitative model. The model has three parts, the regulation of period, amplitude, and output. b. Simulation of the quantitative model. The $\mathrm{x}$-axis represent the concentration of salicylate $\left(\mathrm{I}_{1}\right)$, the y-axis represent the concentration of IPTG $\left(\mathrm{I}_{2}\right)$. Different values of amplitude and period are represented by different colors. The results of experiments are shown in the form of hollow dots with corresponding colors. c. MI metrics between the inputs, amplitude, and period. MI (Amp, T), MI ( $\mathrm{I}_{2}$, Amp), and MI $\left(\mathrm{I}_{1}, \mathrm{~T}\right)$ are close to zero, meaning that the amplitude and period are not coupled and the regulation of the two inputs are orthogonal.

It needs to be noted that the decay of protein is due to cell division $(\delta)$ only, which is different from Tomazou's work [11]. In Tomazou's design, to uncouple the 
degradation of inhibitor proteins and the reporter protein, they need to be degraded by two orthogonal degradation systems. We used the method of removing enzymatic degradation altogether to solve this problem, which is the method used in Potvin's work [10] and is also mentioned in Tomazou's paper on modeling.

For period regulation, we used IPTG to weaken the repression of LacI, which is simpler than Tomazou's method. The main variables were as follows: the gene copy number $\left(p_{1}, p_{2}, p_{3}\right)$; basal transcription rate constant $\left(b_{1}, b_{2}, b_{3}\right)$; maximum transcription rate constant $\left(a_{1}, a_{2}, a_{3}\right)$; character concentration, which describes the amount of repressor required for half-maximal repression rates $\left(k_{1}, k_{2}, k_{3}\right)$; and decay rate of mRNA, which mainly depends on the degradation rate $(\mu)$ for $\mu>>\delta$. The equations for the $m R N A s$ are as follows:

$$
\begin{aligned}
& \frac{d m_{R_{1}}}{d t}=p_{1}\left(b_{1}+a_{1} \frac{k_{3}^{2}}{k_{3}^{2}+R_{3}^{2}}\right)-\mu m_{R_{1}} \\
& \frac{d m_{R_{2}}}{d t}=p_{2}\left(b_{2}+a_{2} \frac{k_{1}^{2}}{k_{1}^{2}+R_{1}^{2}}\right)-\mu m_{R_{2}} \\
& \frac{d m_{R_{3}}}{d t}=p_{3}\left(b_{3}+a_{3} \frac{k_{2}^{2}}{k_{2}^{2}+R_{2}^{2}} f\left(I_{2}\right)\right)-\mu m_{R_{3}}
\end{aligned}
$$

Each equation contains part of the Hill function, and all the Hill coefficients were set to 2. Moreover, the tetR gene was controlled by a promoter without a DNA loop in our design, so we used a linear form equation to describe the inducer effect [18].

$$
f\left(I_{2}\right)=\left\{\begin{array}{cc}
1 & I_{2}<I_{0} \\
\frac{I_{2}}{I_{0}} & I_{2}>I_{0}, \frac{k_{2}^{2}}{k_{2}^{2}+R_{2}^{2}} f\left(I_{2}\right)<1 \\
\frac{k_{2}^{2}+R_{2}^{2}}{k_{2}^{2}} & \frac{k_{2}^{2}}{k_{2}^{2}+R_{2}^{2}} f\left(I_{2}\right) \geq 1
\end{array}\right.
$$

where $I_{0}$ is the characteristic concentration under which the inducer did not work. As for the regulation of amplitude, we used NahR and $\sigma E C F 11$. The corresponding variables were as follows: the gene copy number $\left(p_{4}, p_{e}\right)$, basal transcription rate constant $\left(b_{4}, b_{e}\right)$, maximum transcription rate constant $\left(a_{4}, a_{e}\right)$, and some variables mentioned above $\left(k_{2}, \mu\right)$. The equations for the mRNAs of NahR and $\sigma \mathrm{ECF} 11$ are as 
follows:

$$
\begin{aligned}
& \frac{d m_{R 4}}{d t}=p_{4}\left(b_{4}+a_{4}\right)-\mu m_{R 4} \\
& \frac{d m_{e}}{d t}=p_{e}\left(b_{e}+a_{e} \frac{k_{4}^{2}}{k_{4}^{2}+g\left(R_{4}\right)^{2}}\right)-\mu m_{e}
\end{aligned}
$$

where $g\left(R_{4}\right)$ is the depressor effect of NahR after adding salicylate. This step can be described as follows:

$$
g\left(R_{4}\right)=\frac{k_{R 4}^{2}}{k_{R 4}^{2}+I_{1}^{2}} R_{4}
$$

where $I_{1}$ is the concentration of salicylate. Thus $g\left(R_{4}\right)$ decreases as $I_{1}$ increases, leading to the increase of $d m_{e} / d t$ and, finally, an increased amplitude.

Last but not least, we needed an equation to describe the output, which contained the core of the orthogonal regulation, the dual-input promoter. The dual-input promoter used in our work has been well-studied in our lab [13]. The main variables were as follows: the gene copy number $\left(p_{y}\right)$, basal transcription rate constant $\left(b_{y}\right)$, maximum transcription rate constant $\left(a_{y}\right)$, character concentration $\left(k_{e}\right)$ and some variables mentioned above $\left(k_{3}, \mu\right)$. The equation was as follows:

$$
\frac{d m_{y}}{d t}=p_{y}\left(b_{y}+a_{y} \frac{\left(\frac{E}{k_{e}}\right)^{2}}{1+\left(\frac{E}{k_{e}}\right)^{2}+\left(\frac{R_{3}}{k_{3}}\right)^{2}}\right)-\mu m_{y}
$$

Both $E$ and $R_{3}$ can regulate the transcription of the reporter gene and lead to a change of the fluorescence intensity.

Combining the above equations and the parameters listed in Table S1 and S2, we can obtain the simulation results, as shown in Fig. 4b, in which the average points of the experiments were also shown. It can be seen that the experimental results are generally quantitative and conform to the model, which means the model has a degree of predictability.

On the other hand, to indicate the orthogonality of the model, we calculated the mutual information (MI) between each of the two inputs/outputs [19]. The MI value 
between the two variables $\mathrm{x}$ and $\mathrm{y}$ is defined as:

$$
I[X ; Y]=\iint p(x, y) \ln \frac{p(x, y)}{p(x) p(y)} d x d y
$$

The results were shown in Fig. 4c, which reveals that the regulation is effective, as is the orthogonality.

\section{Discussion}

In this work, we designed and constructed an adjustable repressilator gene circuit and realized orthogonal regulation of its oscillation. This is significant progress after the stabilization of the repressilator gene circuit. Since synthetic repressilator gene circuits have been studied for nearly twenty years, synthetic biologist made great efforts and a lot of progress[20]. At the theoretical level, not only orthogonal regulation[11] but also more complex gene circuits have been designed and simulated [21]. Just because of the solid experimental foundation, which means the mature stable regulatory repressilator genetic circuit, and the guidance of corresponding theory, we can process this work. As an innovation in practice, our work may give some tips to realize the gene circuit at the experiment level. Our work profited much from Potvin's work [10]. Stability is the basis of regulation. Another important point is the choice of components. Quantifiable gene components are conducive to realizing regulation. Last but not least, it is important to appropriately simplify the gene circuit without impacting its function. The method we used to regulate the period is a simplification compared to the design in Tomazou's work. Removing the enzymatic degradation altogether can be seen as a simplification. And it's worth noting that the reporter gene is not the repressor protein itself but regulated by the repressor. This is also a simplification comparing to regulate the repressor protein itself, which is impossible to realize the orthogonal regulation based

on existing theories. So it is an effective method to use the original repressilator to regulate the period and an additional circuit to regulate the amplitude. Thus we can get the orthogonally regulatable repressilator. All these points helped us to complete this work.

Moreover, we also developed a quantitative model to predict the oscillation of the 
circuit under different inducer concentration combinations. This is an important step for realizing modularization of repressilator gene circuits. In previous studies, more attention has been paid to the quality of the oscillation. Therefore, the repressilator always acts as the endpoint of control. However, with the use of a quantitative model, we can make the repressilator modular. The repressilator can be an intermediate node with two inputs and one oscillating output, and this output can regulate other gene circuits that require an oscillating input, such as an oscillating stimulation or activation of gene expression. A modular repressilator can also be used as a biosensor, as mentioned in Tomazou's work [11]. From the analysis of oscillation, we can obtain the quantitative value of two irrelevant inputs.

Still, our design may be difficult to apply because of some other couplings. One example is resource constraints, which are always a concern in synthetic biology [22][23]. Competition for shared cellular resources may have some influence on the regulation of oscillation. It is a new challenge for us to explore the limit of the resource constraints and learn how to deal with them. On the other hand, if the repressilator is used in the fermentation field as an internal regulatory system, environmental stress may be a problem. It is known that the product of fermentation engineering is always poisonous to the engineered bacteria, especially when products pile up [24]. Therefore, repressilator gene circuits are very likely to be influenced in such environments. In this case, exploring methods to avoid this influence, such as by protecting engineered bacteria and separating the product, is also a future research direction.

\section{Methods}

Stains and plasmids. E.coli Top10 was used for plasmid construction, E.coli K-12 DH10B was used for parts characterization, and E.coli DHL708 from Potvin's work was used for circuit measuring throughout this study. PCR, DNA ligation and gibson assembly were used in the construction of the plasmids. The schematic diagrams of detailed cloning steps were shown in Fig. S1 and the plasmids used in this study were listed in Table. S3 
Microfluidic device fabrication. To fabricate the molds for the microfluidic chip, we used a two-layer photolithography method to create SU8 photoresist (Microchem, Japan) patterns with two heights on the silicon wafer; 6-8 $\mathrm{mm}$ thick PDMS was then cast on the silicon wafers and disposed at a curing temperature of $70^{\circ} \mathrm{C}$ for $3 \mathrm{~h}$. Then, the PDMS piece was peeled from the silicon wafer, with the shape of the designed structures transferred to the surface of the PDMS piece. After cleaning with $3 \mathrm{M}$ tape and an oxygen plasma treatment, the PDMS piece was bonded to $0.13 \mathrm{~mm}$ thick glass and heated overnight at $70^{\circ} \mathrm{C}$. Before cell loading, the chip was degassed in vacuum for $10 \mathrm{~min}$

Cell growth and observation. Bacteria were inoculated from single colonies into a 10 $\mathrm{mL}$ glass test tube and then cultured in $\mathrm{LB}$ medium at $37^{\circ} \mathrm{C}$ in a shaker overnight. Then, the cells were diluted 20 -fold with a mixture of $90 \%$ M9 medium and 10\% LB medium for $3 \mathrm{~h}$. Next, the cells were concentrated for 5 min at 4000 r.p.m, resuspended in 100 $\mu \mathrm{L}$ of a $0.1 \%$ BSA solution and loaded into the microfluidic chip. The microfluidic chip was centrifuged for bacteria loading into the trap lines for $10 \mathrm{~min}$ at 4000 r.p.m, after which the chip was connected to our cultivation-observation device. The cultivationobservation setup contained a Nikon Ti-E inverted fluorescence microscope with an EMCCD camera (Andor iXon $\times 3$ DU897) and a CFI plan Apochromat Lambda DM 60× oil immersion objective (NA $1.40 \mathrm{WD} 0.13 \mathrm{~mm}$ ). The microfluidic chip was placed on the motorized microscope stage (with Encoders), and the incubator system temperature was set to $37^{\circ} \mathrm{C}$. Four micro-syringe pumps were used to inject the medium with a flow rate of $40 \mu \mathrm{L} / \mathrm{h}$. Images were acquired every $10 \mathrm{~min}$ for $24 \mathrm{~h}$ or longer. Illumination, exposure time, and camera gain were set to appropriate values and were not changed between experiments.

Media and Buffers. All the chemicals used in the study were purchased from SigmaAldrich unless stated otherwise. LB medium: $10 \mathrm{~g} / \mathrm{L}$ tryptone, $5 \mathrm{~g} / \mathrm{L}$ yeast extract, and $10 \mathrm{~g} / \mathrm{L} \mathrm{NaCl}$. For agar plates, $15 \mathrm{~g} / \mathrm{L}$ agar was added. M9 medium: $6.8 \mathrm{~g} / \mathrm{L} \mathrm{Na}_{2} \mathrm{HPO}_{4}, 3$ $\mathrm{g} / \mathrm{L} \mathrm{KH}_{2} \mathrm{PO}_{4}, 0.5 \mathrm{~g} / \mathrm{L} \mathrm{NaCl}, 1 \mathrm{~g} / \mathrm{L} \mathrm{NH}_{4} \mathrm{Cl}, 0.34 \mathrm{~g} / \mathrm{L}$ thiamine, $0.2 \%$ casamino acids (BD 
Biosciences), $0.4 \%$ glucose, $2 \mathrm{mM} \mathrm{MgSO}_{4}$, and $100 \mu \mathrm{M} \mathrm{CaCl}_{2}$. The $\mathrm{LB}$ medium for overnight culture contained ampicillin and kanamycin at concentrations of $100 \mu \mathrm{g} / \mathrm{mL}$ to maintain the plasmids. The mixed medium for the microfluidic culture contained ampicillin and kanamycin at concentrations of $1 \mu \mathrm{g} / \mathrm{mL}$ at which the cells can grow normally and the plasmids can be maintained.

Data analysis and modeling. For the analysis of microscopic images, we used ImageJ software. Single cells were manually tracked, and the oscillation peaks were found by manually encircling single cells and measuring and comparing the mean fluorescence intensity of each single cell. The periods were calculated by counting the time intervals between two adjacent peaks. The amplitudes were calculated as the difference of the fluorescence intensity between each peak and its nearest trough. The quantitative model was simulated using MATLAB. The parameters of the equations were mainly from previous work[11] and modified to fit our experiment results. The amplitudes and periods were calculated using the "findpeaks" function.

Data availability. We declare that all relevant data supporting the findings of this study are available within the article and its Supplementary Information Files or from the corresponding authors upon request.

\section{Author information}

\section{Corresponding Authors}

*E-mail: pkuluocx@pku.edu.cn.

\section{Author Contributions}

C.L. conceived and supervised the project. F.Z. and C.L. designed the experiment. F.Z., Y.Z. and S.W. performed the experiment. F.Z., Y.S., and W.S. analyzed the data. F.Z. and C.L wrote the manuscript.

\section{Notes}

The authors declare no competing financial interest. 


\section{Acknowledgements}

This study was supported the National Key Research and Development Project (SQ2018YFA090070-03 and 2020YFA0906900) and the NSFC of China (11974002, $11674010)$.

\section{Reference}

[1]Murray, A. W. (1991). Cell cycle extracts. Methods in cell biology, 36, 581-605.

[2]King, K. L., \& Cidlowski, J. A. (1998). Cell cycle regulation and apoptosis. Annual review of physiology, 60(1), 601-617.

[3]Vitaterna, M. H., Takahashi, J. S., \& Turek, F. W. (2001). Overview of circadian rhythms. Alcohol Research \& Health, 25(2), 85.

[4]Laposky, A. D., Bass, J., Kohsaka, A., \& Turek, F. W. (2008). Sleep and circadian rhythms: key components in the regulation of energy metabolism. FEBS letters, 582(1), 142-151.

[5]Rosbash, M., \& Hall, J. C. (1989). The molecular biology of circadian rhythms. Neuron, 3(4), 387-398.

[6]Atkinson, M. R., Savageau, M. A., Myers, J. T., \& Ninfa, A. J. (2003). Development of genetic circuitry exhibiting toggle switch or oscillatory behavior in Escherichia coli. Cell, 113(5), 597-607.

[7]Elowitz, M. B., \& Leibler, S. (2000). A synthetic oscillatory network of transcriptional regulators. Nature, 403(6767), 335-338.

[8]Stricker, J., Cookson, S., Bennett, M. R., Mather, W. H., Tsimring, L. S., \& Hasty, J. (2008). A fast, robust and tunable synthetic gene oscillator. Nature, 456(7221), 516-519. [9]Zhang, Z. B., Wang, Q. Y., Ke, Y. X., Liu, S. Y., Ju, J. Q., Lim, W. A., ... \& Wei, P. (2017). Design of Tunable Oscillatory Dynamics in a Synthetic NF-kB Signaling Circuit. Cell Systems, 5(5), 460-470.

[10]Potvin-Trottier, L., Lord, N. D., Vinnicombe, G., \& Paulsson, J. (2016). Synchronous long-term oscillations in a synthetic gene circuit. Nature, 538(7626), 514517.

[11]Tomazou, M., Barahona, M., Polizzi, K. M., \& Stan, G. B. (2018). Computational 
re-design of synthetic genetic oscillators for independent amplitude and frequency modulation. Cell Systems, 6(4), 508-520.

[12]Goodwin, B. C. (1963). Temporal organization in cells. A dynamic theory of cellular control processes. London,Academic Press,1963. DOI: 10.5962/bhl.title.6268. [13]Zong, Y., Zhang, H. M., Lyu, C., Ji, X., Hou, J., Guo, X., ... \& Lou, C. (2017). Insulated transcriptional elements enable precise design of genetic circuits. Nature communications, 8(1), 1-13.

[14]Wang, P., Robert, L., Pelletier, J., Dang, W. L., Taddei, F., Wright, A., \& Jun, S. (2010). Robust growth of Escherichia coli. Current biology, 20(12), 1099-1103.

[15]Rhodius, V. A., Segall - Shapiro, T. H., Sharon, B. D., Ghodasara, A., Orlova, E., Tabakh, H., ... \& Voigt, C. A. (2013). Design of orthogonal genetic switches based on a crosstalk map of $\sigma \mathrm{s}$, anti - $\sigma \mathrm{s}$, and promoters. Molecular systems biology, 9(1), 702. [16]Schell, M. A., \& Wender, P. E. (1986). Identification of the nahR gene product and nucleotide sequences required for its activation of the sal operon. Journal of bacteriology, 166(1), 9-14.

[17]Xue, H., Shi, H., Yu, Z., He, S., Liu, S., Hou, Y., ... \& Viets, H. (2014). Design, construction, and characterization of a set of biosensors for aromatic compounds. Acs Synthetic Biology, 3(12), 1011.

[18]Choi, P. J., Cai, L., Frieda, K., \& Xie, X. S. (2008). A stochastic single-molecule event triggers phenotype switching of a bacterial cell. Science, 322(5900), 442-446.

[19]Kinney, J. B., \& Atwal, G. S. (2014). Equitability, mutual information, and the maximal information coefficient. Proceedings of the National Academy of Sciences, 111(9), 3354-3359.

[20]Rossi, N. A. , \& Dunlop, M. J. . (2018). Making waves with synthetic oscillators. Cell Systems, 6(4), 406-407.

[21]Perez-Carrasco, R., Barnes, C. P., Schaerli, Y., Isalan, M., Briscoe, J., \& Page, K. M. (2018). Combining a toggle switch and a repressilator within the ac-dc circuit generates distinct dynamical behaviors. Cell systems, 6(4), 521-530.

[22]Weiße, A. Y., Oyarzún, D. A., Danos, V., \& Swain, P. S. (2015). Mechanistic links between cellular trade-offs, gene expression, and growth. Proceedings of the National 
Academy of Sciences, 112(9), E1038-E1047.

[23]Ceroni, F., Algar, R., Stan, G. B., \& Ellis, T. (2015). Quantifying cellular capacity identifies gene expression designs with reduced burden. Nature methods, 12(5), 415418.

[24]Chen, Y., Liu, H., Du, G. C., \& Chen, J. (2007). Effect of 2-bromoethanesulfonate addition on acetate accumulation and variation of bacterial community in anaerobic fermentation of sludge. Chinese journal of applied and environmental biology, 13(1), 108. 


\section{Supplementary Files}

This is a list of supplementary files associated with this preprint. Click to download.

- supportinginformation0531.docx

- nrreportingsummary.pdf 